\title{
Cooperative Game Analysis of a Supply Chain with One Risk-Neutral Supplier and Two Risk-Averse Retailers
}

\author{
Li Changwen ${ }^{1,2}$, Zhou Yongwu ${ }^{1}$, Lu Yong ${ }^{1}$, Guo Jinsen ${ }^{1}$ \\ ${ }^{1}$ School of Business Administration, South China University of Technology, ${ }^{2}$ School of Mathematics Sciences, Huaibei \\ Normal University (China) \\ cwli2008@163.com, sww666@botmail.com, 824046373@.qq.com,512081805@.qq.com
}

Received: December 2013

Accepted: June 2014

\section{Abstract:}

Purpose: This paper considers a two-echelon supply chain composed of one risk-neutral supplier and two risk-averse retailers. The retailers obtain production from the supplier and sell them to the market. Based on the cooperative game theory, the paper studies the appropriate profit allocation of the supply chain when all the players cooperate with each other, where the two retailers face a price-sensitive stochastic demand. The two retailers can either determine their retail prices independently, or decide whether or not to cooperate with each other.

Design/methodology: To allocate the system-wide profit among upstream risk-neutral suppliers and two risk-averse downstream retailers, this paper constructed a cooperative game model, considered as the supermodularity of the characteristic function and the Shapley value of the game.

Findings: By analyzing the construction's cooperative game model, the results show that the profit of the whole supply chain is the highest in the grand coalition structure. This paper also shows that the core of our cooperative game is nonempty, and has the supermodularity property. Based on this, we have computed the Shapley value-based profit allocation for the whole supply chain in a fair manner. 
Originality/value: Although there are a lot of literature examined risk aversion in a supply chain, but they did not consider using cooperative game to study this problem. This the first study is in the context of a supply chain with risk aversion problem.

Keywords: Supply chain, risk-averse, cooperative game, supermodular, Shapley value

\section{Introduction}

Nowadays as the customer requirements vary, the product life cycle is shortening. Thus, managers face more uncertainty and risk (Ma, Liu, Li \& Yan, 2012). This paper considers a two-echelon supply chain composed of one risk-neutral supplier and two risk-averse retailers. The retailers obtain production from the supplier and sell them to the market. We are interested in analyzing profit allocations when the retailers are risking aversion under the players cooperating with each other. Here we assume that each retailer is able to decide on whether it will trade with the supplier in a non-cooperative setting or in a cooperative setting. In the non-cooperative setting, the supplier and the retailers make their wholesale prices and retail prices in Stackelberg equilibrium respectively. In the cooperative setting, the retailers choose the globally-optimal retail prices that maximize the system-wide profit. As Leng and Parlar (2012) have stated, there are a lot of practical examples showing that the players "are" free to decide on whether or not cooperate with each other. For example, Ford Motor Company's Struandale engine plant in South Africa (an upstream player) supplies the Duratorq TDCi diesel engines to the firm's global assembly plants(multiple downstream players) which manufactures the Ford Ranger pick-up trucks(Leng \& Parlar, 2012). Those assembly plants are actually free to decide on whether they can buy from the plant in South Africa at negotiated transfer prices in the cooperative setting or at non-cooperative (Stackelberg equilibrium) transfer prices. So it is natural to adopt a cooperative game model to study these issues.

Coordinating supply chains have been a major issue in supply chain management (SCM) research. Much of this research has assumed that the agents in the supply chain are risk neutral; that is to say, they maximize their respective expected profits. Many well-known contract types are identified, including buy-back, revenue-sharing, quantity flexibility, sales rebate, and quantity discount; which have been shown to coordinate the supply chain in the risk neutral case, these literature have been surveyed by Cachon (2003). In reality, however, the uncertainty of market demand usually brings certain risk to the gains of supply chain member enterprises. Members with different risk attitudes are different towards the market yeild risk. For instance, for fear of risks, the chain member enterprises with risk aversion characteristics often choose relatively conservative decision-making practice. In contrast, member companies with risk preferences usually chase high risk and high yield. Therefore, risk 
attitudes of member enterprises should be taken fully into account in the design of supply chain coordination contract.

Our research is related to the existing literature in two ways. Firstly, our research is related to the operational risk averse literature. Secondly, it is related to the cooperative game mode in operational literature.

Eeckhoudt, Gollier and Schlesinger (1995) studied the mechanism design of coordination contract in supply chain which contains a single risk aversion partner. Agrawal and Seshadri (2000) considered the combined optimization decision of inventory and the price for riskaversion members. Tsay (2002) studied the situation in which both suppliers and retailers are risk aversive and made analysis when counter purchase is nested in the model. Chen and Federgruen (2000) studied the newsvendor problem using the mean-variance framework. They exhibit how a systematic mean-variance trade-off analysis can be carried out efficiently, and how the resulting strategies differ from those obtained in the standard analyses. Choi, Li, Yan and Chiu (2008) investigated the issues of channel coordination in a supply chain when individual supply chain decision makers take the mean-variance objective. Hsieh and Lu (2010) studied the manufacturer's return policy and the retailers' decisions in a supply chain consisting of one manufacturer and two risk-averse retailers under a single-period setting with price-sensitive random demand. Ma, Liu, Li \& Yan (2012) considered a supply chain with one manufacturer and one retailer where there is only one product with stochastic demand. Xu, Dan, Zhang and Liu (2014) investigated the impact of establishing a dual-channel supply chain coordinating contract when the supply chain agents are risk aversion under a mean-variance model. Kim \& Park (2014) analyzed risk management contracts used to handle currency risk in a decentralized supply chain that consisting of risk-averse divisions in a multinational firm. However, for those papers mentioned, none of them has considered the cooperative game problem.

On the other hand, papers employing cooperative game theory to study supply chain management are becoming more popular. Gerchak and Gupta (1991) modeled their inventory problem as a cooperative game and analyzed the joint cost allocation problem. Rosenthal (2008) developed a cooperative game that provides transfer prices for the intermediate products in a vertically integrated supply chain, in which the divisions share technology and transactions costs. Granot and Sosic (2005) study a multi-stage model of decentralized distribution system with inventory sharing consisting of $\mathrm{n}$ retailers. In the cooperative stage, they show that Shapley value encourages the retailers to share all of their residuals. Guo, Leng and Wang (2012) considered two game-theoretic settings to determine the optimal values of an issuer's interchange fee rate, an acquirer's merchant discount rate and a merchant's retail prices for multiple products in a credit card network. Yu, Yang, Xing and Li (2014) presented an improved genetic annealing algorithm to improve the efficiency of information shared among the innovation agents of customer collaborative product innovation. They model a 
cooperative game problem and focus on the coalition of the supply chain partners. Finally, these papers have not considered risk-averse behavior of the supply chain agents.

The work most closely related to ours is those of Leng and Parlar (2012). Leng and Parlar (2012), which have considered the transfer pricing decision for a multidivisional firm with an upstream division and multiple downstream divisions. To allocate the firm-wide profit between upstream and downstream divisions, they construct a cooperative game and compute the Shapley value-based transfer prices for the firm. However, we assume the retailers have risk attitude. In addition, the demand in this paper is stochastic which is different from the literature of Leng and Parlar (2012).

This paper considers a two-echelon supply chain composed of one risk-neutral supplier and two risk-averse retailers under a mean-variance model. We will use cooperative game theory to allocate profit to assure that the retailers are better (by achieving more profits) in the cooperative setting than in the non-cooperative setting. We would also prove that our cooperative game is supermodular, and that the game have a nonempty core, that is, we can find a fair allocation for the supplier and retailers in cooperative setting. So our contribution is that we have considered a risk-averse supply chain using a cooperative game, which is not considered before.

We will present our model and results in the next four sections. Section 2 gives the problem description and some notations. We consider the different coalition structures in Section 3. In Section 4, we formulate a three-player cooperative model and show the supermodular of the game, and then allocated the profit using the Shapley value. Finally, some conclusions are made in Section 5.

\section{Problem Description and Some Notations}

Consider a two-echelon supply chain with a risk-neutral supplier selling a single product at a wholesale price to two risk-averse retailers who in turn sell the product to the consumer market. We assume that the demand function of retailer $i$ is $q_{i}=\alpha_{i}-\beta_{i} p_{i}$, where $\alpha_{i}$ is the stochastic market base for retailer $i$ with mean $\alpha_{i}$, variance $\sigma^{2}$ for both retailers, $p_{i}$ is the retail price of retailer $i$. Considering the risk sensitivity of the retailers, we assume that each retailer assesses his utility via the following Mean-Variance value function of his random profit:

$$
U\left(\pi_{i}\right)=\left(\alpha_{i}-\beta_{i} p_{i}\right)\left(p_{i}-w_{i}\right)-\eta_{i}\left(p_{i}-w_{i}\right)^{2} \sigma^{2}, i=1,2
$$

where $w_{i}$ is the unit wholesale price of the supplier; $\eta_{i}$ is the constant absolute risk aversion of retailer $i$, which is defined in the Arrow-Pratt sense, $\eta_{i} \geq 0$; the risk-neutral supplier' utility function is: 


$$
U\left(\pi_{0}\right)=E\left(\pi_{0}\right)=\sum_{i=1}^{2}\left(\alpha_{i}-\beta_{i} p_{i}\right)\left(w_{i}-c\right)
$$

Where $c$ is the unit production cost of the supplier.

For expositional simplicity, we denote supplier as 0 , the two retailers as 1 and 2 respectively. Let $N=\{0,1,2\}$ be the set of all players including one supplier and two retailers. A subset $Z \subseteq N$ is called a coalition, and the set $N$ of all players is referred to as the grand coalition. Any partition of $N$, i.e., $L=\left\{Z_{1}, Z_{2}, \ldots, Z_{k}\right\}$ where $k \leq 3, \cup_{i-1}^{k} Z_{i}=N, Z_{i} \cap Z_{j}=\varnothing, i \neq j$ corresponds to a coalition structure $L$. We also assume that in the non-cooperative setting where the supplier acts as the leader and the retailers that are not in coalition act as the followers and choose the wholesale prices in Stackelberg equilibrium.

\section{Cooperative Game Model}

We now use cooperative game theory to solve the allocation of profit. Following von Neumann and Morgenstern (1944), we first construct a cooperative game in characteristic-function form by computing the characteristic value of each possible coalition, which is defined as the utility of the coalition. Then we analyze the stability of core and consider the fair allocation of profit using Shapley value. The alliances of the supply chain system have the following several forms: one-person coalition $\{\{0\},\{1\},\{2\}\}$, two-person coalitions $\{\{01\},\{2\}\},\{\{02\},\{1\}\}$, $\{\{0\},\{12\}\}$, and grand coalition $\{\{12\}\}$. To calculate characteristic value, we first give the utility under different coalition structures.

\subsection{The Utility under Different Coalition Structures}

\subsubsection{One-Person Coalition $\{\{0\},\{1\},\{2\}\}$}

When any player does not cooperate with another player in the supply chain system, then oneperson coalition $\{\{0\},\{1\},\{2\}\}$ is formed. In this setting, the supplier acts as the Stackelbergleaders, first makes a decision about the wholesale prices $w_{i}^{0}(i=1,2)$, the two retailers act as the Stackelberg-followers, then decide the retail prices $p^{0}{ }_{i}(i=1,2)$, so the optimization problem can be modeled as:

$$
\left\{\underset{p_{i}}{\operatorname{Max}} U\left(\pi_{0}^{0}\right)=\sum_{i=1}^{2}\left(\alpha_{i}-\beta_{i} p_{i}\right)\left(w_{i}-c\right), \text { s.t. } \operatorname{Max}_{w_{i}} U\left(\pi_{i}^{0}\right)=\left(\alpha_{i}-\beta_{i} p_{i}\right)\left(p_{i}-w_{i}\right)-\eta_{i}\left(p_{i}-w_{i}\right)^{2} \sigma^{2}, i=1,2,\right.
$$

Using backward induction method to solve the above optimization problem, the equilibrium solution can be obtained as: 


$$
w_{i}^{0}=\frac{\alpha_{i}+\beta_{i} c}{2 \beta_{i}}, p_{i}^{0}=\frac{2 \alpha_{i} \beta_{i}+\left(\beta_{i}+2 \eta_{i} \sigma^{2}\right)\left(\alpha_{i}+\beta_{i} c\right)}{4 \beta_{i}\left(\beta_{i}+\eta_{i} \sigma^{2}\right)} .
$$

The utility of two retailers and supplier can respectively be obtained as:

$$
U\left(\pi_{\mathrm{i}}^{0}\right)=\frac{1}{16\left(\beta_{i}+\eta_{i} \sigma^{2}\right)}\left(\alpha_{i}-\beta_{i} c\right)^{2}, i=1,2, U\left(\pi_{0}^{0}\right)=\sum_{i=1}^{2} \frac{\beta_{i}+2 \eta_{i} \sigma^{2}}{8 \beta_{i}\left(\beta_{i}+\eta_{i} \sigma^{2}\right)}\left(\alpha_{i}-\beta_{i} c\right)^{2}
$$

Then the total utility of the whole the supply chain system is:

$$
U\left(\pi^{0}\right)=\sum_{i=1}^{3} U\left(\pi_{i}^{0}\right)=\sum_{i=1}^{2} \frac{3 \beta_{i}+4 \eta_{i} \sigma^{2}}{16 \beta_{i}\left(\beta_{i}+\eta_{i} \sigma^{2}\right)}\left(\alpha_{i}-\beta_{i} c\right)^{2}
$$

From the above results under one-person coalition $\{\{0\},\{1\},\{2\}\}$, we can see that the utility of two retailers decreases as $\eta_{i}$ increases, but the utility of the supplier increases as $\eta_{i}$ increases, and the total utility of the whole the supply chain system increases as $\eta_{i}$ increases.

\subsubsection{Two-Person Coalition $\{\{01\},\{2\}\}$}

When the supplier 0 cooperates with retailer 1 to form a coalition $\{01\}$, coalition $\{01\}$ as the Stackelberg-leaders, first makes a decision about the wholesale $w_{i}^{0}(i=1,2)$ and the retail price $p_{1}^{01}$, the retailer 2 as the Stackelberg-followers, then decide the retail price $p_{2}^{01}$, so the optimization problem can be modeled as:

$$
\left\{\begin{array}{c}
\underset{p_{1}, w_{1}, w_{2}}{\operatorname{Max}} U\left(\pi_{01}^{01}\right)=\left(\alpha_{1}-\beta_{1} p_{1}\right)\left(p_{1}-c\right)-\eta_{1}\left(p_{1}-w_{1}\right)^{2} \sigma^{2}+\left(\alpha_{2}-\beta_{2} p_{2}\right)\left(w_{2}-c\right) \\
\text { s.t.Max } U\left(\pi_{2}^{01}\right)=\left(\alpha_{2}-\beta_{2} p_{2}\right)\left(p_{2}-w_{2}\right)-\eta_{2}\left(p_{2}-w_{2}\right)^{2} \sigma^{2}
\end{array}\right.
$$

Using backward induction method, we can get:

$$
p_{2}^{01}=\frac{\alpha_{2}+\left(\beta_{2}+2 \eta_{2} \sigma^{2}\right) w_{2}^{01}}{2\left(\beta_{2}+\eta_{2} \sigma^{2}\right)}
$$

Substituting $p_{2}^{01}$ into $U\left(\pi_{01}^{01}\right)$, we know that coalition $\{01\}$ jointly determines retail price $p_{1}^{01}$ and wholesale prices $w_{i}^{0}(i=1,2)$ to maximize the utility function $U\left(\pi_{01}^{01}\right)$. The Hessian matrix of $U\left(\pi_{01}^{01}\right)$ is:

$$
H=\left(\begin{array}{ccc}
-2 \beta_{1}-2 \eta_{1} \sigma^{2} & 2 \eta_{1} \sigma^{2} & 0 \\
2 \eta_{1} \sigma^{2} & -2 \eta_{1} \sigma^{2} & 0 \\
0 & 0 & -2 \beta_{2} \frac{\alpha_{2}+\beta_{2}+2 \eta_{2} \sigma^{2}}{2\left(\beta_{2}+\eta_{2} \sigma^{2}\right)}
\end{array}\right)
$$


We can find that $H$ is negatively definited, so the utility function $U\left(\pi_{01}^{01}\right)$ is a concave function on $\left(p_{2}^{01}, w_{1}^{0}, w_{2}^{0}\right)$, the unique equilibrium solution is:

$$
p_{1}^{01}=w_{1}^{01}=\frac{\alpha_{1}+\beta_{1} c}{2 \beta_{1}}, w_{2}^{01}=\frac{\alpha_{2}+\beta_{2} c}{2 \beta_{2}}, p_{2}^{01}=\frac{2 \alpha_{2} \beta_{2}+\left(\beta_{2}+2 \eta_{2} \sigma^{2}\right)\left(\alpha_{2}+\beta_{2} c\right)}{4 \beta_{2}\left(\beta_{2}+\eta_{2} \sigma^{2}\right)}
$$

Then the utility of coalition $\{01\}$ and the retailer 2 are:

$$
U\left(\pi_{01}^{01}\right)=\frac{1}{4 \beta_{1}}\left(\alpha_{1}-\beta_{1} c\right)^{2}+\frac{\beta_{2}+2 \eta_{2} \sigma^{2}}{8 \beta_{2}\left(\beta_{2}+\eta_{2} \sigma^{2}\right)}\left(\alpha_{2}-\beta_{2} c\right)^{2}, U\left(\pi_{2}^{01}\right)=\frac{1}{16\left(\beta_{2}+\eta_{2} \sigma^{2}\right)}\left(\alpha_{2}-\beta_{2} c\right)^{2}
$$

The total utility of the whole supply chain system is:

$$
U\left(\pi^{01}\right)=U\left(\pi_{01}^{01}\right)+U\left(\pi_{2}^{01}\right)=\frac{1}{4 \beta_{1}}\left(\alpha_{1}-\beta_{1} c\right)^{2}+\frac{3 \beta_{2}+4 \eta_{2} \sigma^{2}}{16 \beta_{2}\left(\beta_{2}+\eta_{2} \sigma^{2}\right)}\left(\alpha_{2}-\beta_{2} c\right)^{2}
$$

From the above results under two-person coalition $\{\{01\},\{2\}\}$, we can see that the utility of coalition $\{01\}$ increases as $\eta_{i}$ increases, but the utility of the retailer 1 decreases as $\eta_{i}$ increases, and the utility of the whole supply chain system increases as $\eta_{i}$ increases.

\subsubsection{Two-Person Coalition $\{\{02\},\{1\}\}$}

When the supplier 0 cooperate with retailer 2 to form an alliance $\{02\}$, coalition $\{02\}$ as the Stackelberg-leaders, the retailer 1 as the Stackelberg-followers, this is similar with two-person coalition $\{\{02\},\{1\}\}$, so we can get the utility of coalition $\{02\}$ and the retailer 1 are respectively as follows:

$$
U\left(\pi_{02}^{02}\right)=\frac{1}{4 \beta_{2}}\left(\alpha_{2}-\beta_{2} c\right)^{2}+\frac{\beta_{1}+2 \eta_{1} \sigma^{2}}{8 \beta_{1}\left(\beta_{1}+\eta_{1} \sigma^{2}\right)}\left(\alpha_{1}-\beta_{1} c\right)^{2}, U\left(\pi_{1}^{02}\right)=\frac{1}{16\left(\beta_{1}+\eta_{1} \sigma^{2}\right)}\left(\alpha_{1}-\beta_{1} c\right)^{2}
$$

Then the total utility of the whole supply chain system is

$$
U\left(\pi^{02}\right)=U\left(\pi_{02}^{02}\right)+U\left(\pi_{1}^{02}\right)=\frac{1}{4 \beta_{2}}\left(\alpha_{2}-\beta_{2} c\right)^{2}+\frac{3 \beta_{1}+4 \eta_{1} \sigma^{2}}{16 \beta_{1}\left(\beta_{1}+\eta_{1} \sigma^{2}\right)}\left(\alpha_{1}-\beta_{1} c\right)^{2}
$$

From the above results under two-person coalition $\{\{02\},\{1\}\}$, we can see that the utility of coalition $\{02\}$ increases as $\eta_{i}$ increases, but the utility of the retailer 1 decreases as $\eta_{i}$ increases, and the total utility of the whole supply chain system increases as $\eta_{i}$ increases. 


\subsubsection{Two-Person Coalition $\{\{0\},\{12\}\}$}

When the retailer 1 cooperates with retailer 2 to form an alliance $\{12\}$, coalition $\{12\}$ as the Stackelberg-followers, the supplier 0 still as the Stackelberg-leaders, in this setting, the equilibrium solution is same as the one-person coalition $\{\{0\},\{1\},\{2\}\}$, so we can get the utility of coalition $\{12\}$ and the supplier 0 are respectively as follows:

$$
U\left(\pi_{12}^{12}\right)=\sum_{i=1}^{2} \frac{1}{16\left(\beta_{i}+\eta_{i} \sigma^{2}\right)}\left(\alpha_{i}-\beta_{i} c\right)^{2}, U\left(\pi_{0}^{12}\right)=\sum_{i=1}^{2} \frac{\beta_{i}+2 \eta_{i} \sigma^{2}}{8 \beta_{i}\left(\beta_{i}+\eta_{i} \sigma^{2}\right)}\left(\alpha_{i}-\beta_{i} c\right)^{2}
$$

Then the total utility of the whole supply chain system is:

$$
U\left(\pi^{12}\right)=U\left(\pi_{12}^{12}\right)+U\left(\pi_{0}^{12}\right)=\sum_{i=1}^{2} \frac{3 \beta_{i}+4 \eta_{i} \sigma^{2}}{16 \beta_{i}\left(\beta_{i}+\eta_{i} \sigma^{2}\right)}\left(\alpha_{i}-\beta_{i} c\right)^{2}
$$

From the above results under two-person coalition $\{\{0\},\{12\}\}$, we can see that the utility of coalition $\{12\}$ decreases as $\eta_{i}$ increases, but the utility of the supplier 0 increases as $\eta_{i}$ increases, and the total utility of the whole supply chain system increases as $\eta_{i}$ increases.

\subsubsection{Grand Coalition $\{012\}$}

When the retailers cooperate with the supplier to form grand coalition $\{012\}$, the grand coalition $\{012\}$ jointly determines the globally-optimal retail price $p_{1}^{01}$ and wholesale $w_{i}^{0}(i=$ $1,2)$ to maximize the utility function $U\left(\pi^{012}\right)$ :

$$
\operatorname{Max}_{p_{1}, p_{2}, w_{1}, w_{2}} U\left(\pi^{012}\right)=\sum_{i=1}^{2}\left[\left(\alpha_{i}-\beta_{i} p_{i}\right)\left(p_{i}-c\right)-\eta_{i}\left(p_{i}-w_{i}\right)^{2} \sigma^{2}\right]
$$

It is easy to prove that the Hessian matrix of $U\left(\pi^{012}\right)$ is negatively definited, so the unique equilibrium solution is:

$$
p_{i}^{012}=w_{i}^{012}=\frac{\alpha_{i}+\beta_{i} c}{2 \beta_{i}}, i=1,2
$$

Then the utility of coalition $\{012\}$ is:

$$
U\left(\pi^{012}\right)=\sum_{i=1}^{2} \frac{1}{4 \beta_{i}}\left(\alpha_{i}-\beta_{i} c\right)^{2}
$$

Under the grand coalition $\{012\}$, we find that the utility of coalition $\{012\}$ has noting to do with the risk aversion sensitivity $\eta_{i}$, this is different from the other coalition structures we have analyzed. 


\subsection{The Characteristic Values of Different Coalitions}

Through the analysis of the different alliance structure, we find that

$$
U\left(\pi^{012}\right)>U\left(\pi^{01}\right)\left(U\left(\pi^{02}\right)\right)>U\left(\pi^{12}\right)=U\left(\pi^{0}\right)
$$

From (14), we can see that the total utility of the whole supply chain achieves the maximum in the grand coalition. We adopt the method of cooperative game to allocate the profit under the grand coalition for the supply chain system. Here we first show the characteristic values of three-player cooperative game $(N, v)$, where $N=\{0,1,2\}$, and define the characteristic values as follows: $v(\varnothing)=0, v(\{0\})=U\left(\pi_{0}^{0}\right), v(\{1\})=U\left(\pi_{1}^{0}\right), v(\{2\})=U\left(\pi_{2}^{0}\right), v(\{12\})=U\left(\pi_{12}^{12}\right), v(\{01\})$ $=U\left(\pi_{01}^{01}\right), v(\{02\})=U\left(\pi_{02}^{02}\right), v(\{12\})=U\left(\pi^{12}\right)$.

\section{The Stability of the Grand Coalition}

We use the solution concepts of Shapley value to find a fair scheme of allocating the profit $U\left(\pi^{012}\right)$ among the three players. Before we allocated the profit, we first investigate whether our cooperative game in the characteristic-function form is superadditive and convex. A threeplayer cooperative game $G$ is superadditive if $v\left(S_{1} \cup S_{2}\right) \geq v\left(S_{1}\right)+\left(S_{2}\right)$ for any two disjoint coalitions $S_{1}$ and $S_{2}$ (Straffin, 1993). Moreover, the game is convex and superadditive if its characteristic function is supermodular (Driessen, 1988). For our problem, the characteristic function of our three-player cooperative game is supermodular (Guo, Leng \& Wang,2012) if

$$
v\left(S_{2} \cup\{i\}\right)-v\left(S_{2}\right) \geq v\left(S_{1} \cup\{i\}\right)-v\left(S_{1}\right), S_{1} \subseteq S_{2} \subseteq N \backslash\{i\} .
$$

Theorem 1: The characteristic function of three-player cooperative game $(N, v)$ is supermodular. So the game is superadditive and convex.

Proof:

We need to prove that

$$
v\left(S_{2} \cup\{i\}\right)-v\left(S_{2}\right) \geq v\left(S_{1} \cup\{i\}\right)-v\left(S_{1}\right), S_{1} \subseteq S_{2} \subseteq N \backslash\{i\}, i=0,1,2
$$

If $S_{1}=S_{2}$, then the above condition for the supermodularity must be satisfied. Next we assume that $S_{1} \subseteq S_{2} \subseteq N \backslash\{i\}$, and examine that if the above condition is satisfied.

1. If $S_{1}=\varnothing$ and $S_{2}=0$, then player $i$ is either 1 or 2 , and we find that

$$
v(\{0\}) \cup(\{i\})-v(\{0\}) \geq v(\{i\})-v(\varnothing), i=1,2
$$


Because for $i=1,2$,

$$
\begin{gathered}
v(\{0 i\})=\frac{1}{4 \beta_{i}}\left(\alpha_{i}-\beta_{i} c\right)^{2}+\frac{\beta_{3-i}+2 \eta_{3-i} \sigma^{2}}{8 \beta_{3-i}\left(\beta_{3-i}+\eta_{3-i} \sigma^{2}\right)}\left(\alpha_{3-i}-\beta_{3-i} c\right)^{2} \\
>v(\{0\})+v(\{i\})=\frac{3 \beta_{i}+4 \eta_{i} \sigma^{2}}{16\left(\beta_{i}+\eta_{i} \sigma^{2}\right)}\left(\alpha_{i}-\beta_{i} c\right)^{2}+\frac{\beta_{3-i}+2 \eta_{3-i} \sigma^{2}}{8 \beta_{3-i}\left(\beta_{3-i}+\eta_{3-i} \sigma^{2}\right)}\left(\alpha_{3-i}-\beta_{3-i} c\right)^{2}
\end{gathered}
$$

Therefore, the supermodularity condition is satisfied.

2. If $S_{1}=\varnothing$ and $S_{2}=1$, then player $i$ is either 0 or 2 . If $i=2$, then

$$
v(\{1\} \cup\{2\})-v(\{1\}) \geq v(\{2\})-v(\varnothing)
$$

Because

$$
V(\{1\} \cup\{2\})=U\left(\pi_{12}^{12}\right)=U\left(\pi_{1}^{0}\right)+U\left(\pi_{2}^{0}\right)=V(\{1\})+V(\{2\})
$$

If $i=0$, then

$$
\boldsymbol{v}(\{\mathbf{1}\} \cup\{0\})-\boldsymbol{v}(\{\mathbf{1}\}) \geq(\{0\})-\boldsymbol{v}(\varnothing)
$$

Therefore, the supermodularity condition is satisfied.

3. If $S_{1}=\varnothing$ and $S_{2}=2$, then player $i$ is either 0 or 1 . Similar to case 2 , we find that the supermodularity condition is satisfied.

4. If $S_{1}=0$ and $S_{2}=01$, then player $i$ is 2 . We have

$$
v(\{01\} \cup\{2\})-v(\{01\}) \geq(\{02\})-v(\{0\})
$$

Because

$$
\begin{gathered}
V(\{01\} \cup\{2\})-v(\{01\})=U\left(\pi^{012}\right)-U\left(\pi_{01}^{01}\right)=\frac{1}{8\left(\beta_{2}+\eta_{2} \sigma^{2}\right)}\left(\alpha_{2}-\beta_{2} c\right)^{2} \\
V(\{02\})-v(\{0\})=U\left(\pi_{02}^{02}\right)-U\left(\pi_{0}^{0}\right)=\frac{1}{8\left(\beta_{2}+\eta_{2} \sigma^{2}\right)}\left(\alpha_{2}-\beta_{2} c\right)^{2}
\end{gathered}
$$

Therefore, the supermodularity condition is satisfied.

5. If $S_{1}=0$ and $S_{2}=02$, then player $i$ is 1 . Similar to case 2 , we can easily find that the supermodularity condition is satisfied.

6. If $S_{1}=1$ and $S_{2}=12$, then player $i$ is 0 , we have

$$
v(\{0\}) \cup(\{12\})-v(\{12\}) \geq v(\{10\})-v(\{1\})
$$

Because

$$
v(\{0\}) \cup(\{12\})-v(\{12\})=U\left(\pi^{012}\right)-U\left(\pi_{12}^{12}\right)=\sum_{i=1}^{2} \frac{3 \beta_{i}+4 \eta_{i} \sigma^{2}}{16\left(\beta_{i}+\eta_{i} \sigma^{2}\right)}\left(\alpha_{i}-\beta_{i} c\right)^{2}
$$




$$
v(\{10\})-v(\{1\})=U\left(\pi_{01}^{01}\right)-U\left(\pi_{1}^{0}\right)=\frac{3 \beta_{1}+4 \eta_{1} \sigma^{2}}{16\left(\beta_{1}+\eta_{1} \sigma^{2}\right)}\left(\alpha_{1}-\beta_{1} c\right)^{2}+\frac{\beta_{2}+2 \eta_{2} \sigma^{2}}{8 \beta_{2}\left(\beta_{2}+\eta_{2} \sigma^{2}\right)}\left(\alpha_{2}-\beta_{2} c\right)^{2}
$$

Therefore, the supermodularity condition is satisfied.

7. If $S_{1}=1$ and $S_{2}=01$, then player $i$ is 2 , we have

$$
v(\{0\}) \cup(\{12\})-v(\{01\}) \geq v(\{12\})-v(\{1\})
$$

Therefore, the supermodularity condition is satisfied.

8. If $S_{1}=2$ and $S_{2}=, 12$, then player $i$ is 0 , similar to case 6 , we find that the supermodularity condition is satisfied.

9. I f $S_{1}=2$ and $S_{2}=02$, then player $i$ is 1 , similar to case 7 , we find that the supermodularity condition is satisfied.

In conclusion, the characteristic function of this game is supermodular, and thus, the game is convex and superadditive.

As Theorem 1 implies, when members of the supply chain form a grand coalition, the characteristic value of the coalition is higher. It thus follows that three players have the incentive to join the grand coalition $\{12\}$. This means that the grand coalition $\{12\}$ is stable if there exists a set of fair allocation schemes. Letting $y_{i}$ denote the profit allocated to player $i$, $i \in\{0,1,2\}$, we call a proper allocation scheme $\left(y_{0}, y_{1}, y_{2}\right)$ imputation for our game $G=(N, v)$ if the scheme satisfies the following two properties (Driessen,1988):

- individual rationality, that is, $y_{i} \geq v(\{i\}), i=0,1,2$

- collective rationality, that is, $\sum_{i=1}^{3} y_{i}=v(\{012\})$. The core is the set of all undominated imputations (fair allocation schemes) $\left(y_{0}, y_{1}, y_{2}\right)$ such that $\sum_{i \in S} y_{i} \geq V(\{S\})$ for all coalitions $S \subseteq N$.

Corollary 1: The core of three-player cooperative game $(N, v)$ is nonempty.

Proof:

From Theorem 1 we can conclude that the game $(N, v)$ is convex game, which have a very good property is that the core of convex game is nonempty. This corollary is thus proved.

As the above theorem indicates, any point in the nonempty core represents a fair allocation scheme. So the core includes many allocation schemes rather than only a unique scheme, To 
find a unique allocation solution, we next focus on the fair allocation in terms of the Shapley value (Shapley, 1953). Shapley value are the most used solutions in cooperative game theory. For an ${ }^{n-p l a y e r ~ c o o p e r a t i v e ~ g a m e, ~ t h e ~ S h a p l e y ~ v a l u e ~ f o r ~ p l a y e r ~} i$ is computed as:

$$
\Phi_{i}(v)=\sum_{S \subseteq N \backslash i} \frac{|S| !(n-|S|-1) !}{n !}(v(S \cup\{i\})-v(S))
$$

where $\phi_{i}(v)$ is the Shapley value of player $i,|S|$ is the players' number in the coalition $S, n$ is all players' numbers, and $v(S)$ is the value function of the coalition $S$. The marginal contribution of adding player ii to coalition $S$ is $v(S \cup\{i\})-v(S)$. According to the definition, the Shapley value is obtained by averaging the marginal contributions for all the coalitions of the game players (Shapley, 1971).

Using the above formula, we can write the unique allocation scheme in terms of Shapley value for our cooperative game $(N, v)$ as,

$$
\begin{aligned}
& \phi_{0}(v)=\frac{1}{6}[2 v(012)+2 v(0)+v(01)+v(02)-v(1)-v(2)-2 v(12)] \\
& \phi_{1}(v)=\frac{1}{6}[2 v(012)+2 v(1)+v(01)+v(12)-v(0)-v(2)-2 v(02)] \\
& \phi_{2}(v)=\frac{1}{6}[2 v(012)+2 v(2)+v(02)+v(12)-v(0)-v(1)-2 v(01)]
\end{aligned}
$$

Substituting the characteristic value of three-player cooperative game $(N, v)$ into $(29-31)$, we can get the following corollary.

Corollary 2: For our three-player cooperative game $(N, v)$, the Shapley value $\phi_{i}(v)$ is calculated as:

$$
\Phi_{0}(v)=\sum_{i=1}^{2} \frac{5 \beta_{i}+8 \eta_{i} \sigma^{2}}{32 \beta_{i}\left(\beta_{i}+\eta_{i} \sigma^{2}\right)}\left(\alpha_{i}-\beta_{i} c\right)^{2}, \Phi_{1}(v)=\frac{3 \beta_{i}}{32 \beta_{i}\left(\beta_{i}+\eta_{i} \sigma^{2}\right)}\left(\alpha_{i}-\beta_{i} c\right)^{2}, i=1,2
$$

From the conclusion of corollary 2 , we can see that as $\eta_{i}$ becomes smaller, the retailer $i$ gets more profit based on Shapley value allocation, especially when $\eta_{i}=0$ the retailer $i$ becomes a risk-neutral retailer, then retailer can gets the largest profit $\phi_{i}(v)=\frac{3}{32}\left(\alpha_{i}-\beta_{i} c\right)^{2}$. But as $\eta_{i}$ becomes smaller, the supplier gets less profit. Especially when $\eta_{i}=0$, the supplier can gets the least profit $\Phi_{0}(v)=\sum_{i=1}^{2} \frac{5}{32 \beta_{i}}\left(\alpha_{i}-\beta_{i} c\right)^{2}$, when $\eta_{i} \rightarrow \infty$, the supplier can gets the largest profit $\Phi_{0}(v)=\sum_{i=1}^{2} \frac{1}{4 \beta_{i}}\left(\alpha_{i}-\beta_{i} c\right)^{2}$ 


\section{Conclusion}

This paper studies a two-echelon supply chain with one risk-neutral supplier and two riskaverse retailers. A cooperative game approach is used to allocate the profit of the supply chain system in the grand coalition. To find the characteristic function of the game, we first considered the optimal pricing of the risk-averse retailers in non-cooperative setting. In this setting, the coalitions including supplier act as the Stackelberg-leaders, and the retailer out of the coalition acts as the Stackelberg-followers. The Stackelberg equilibrium solutions are computed for different coalitions to define the characteristic value of the cooperative game. We also concluded that the core of the cooperative game is nonempty through showing the supermodularity of the characteristic function. Then, we computed Shapley value for our game to find a unique, fair allocation scheme, which is in the core because of the convexity of our game. Thus, the allocation scheme suggested by the Shapley value can assure the stability of the grand coalition.

This paper makes the following contributions to the operations management literature: first, we not only address the cooperative game problem caused by the players of the supply chain but also provide insights into the conflicts of risk aversion. Second, we found that risk aversion strongly influences the manufacturer and the retailer's pricing strategies and expected profits under different coalition structures. Our studies show that in cooperative setting, based on the Shapley value allocation, the risk-averse retailer gets a lower profit and the risk-neutral supplier gets a higher profit as risk intensity increases.

Our analysis also might have some limitations. We only use Mean-variance analysis model risk aversion of the retailers. However, there are two other important risk measures widely used in finance field, that is, the Value-at-Risk (VaR), and the CVaR. Therefore, we may need to explore the risk aversion model. We consider a single retailer; however, it might be interesting to study multiple retailers cooperating with each other.

\section{Acknowledgment}

The authors would like to express their thanks to the Editor Dr. Mincong Tang and two anonymous referees for their helpful comments and valuable suggestions. This work was partly supported by the Natural Science Foundation of China (71131003, 71371075), the Social Science Foundation of China (12CGL041), the Anhui Provincial Natural Science Foundation (KJ2013A235). 


\section{References}

Agrawal, V., \& Seshadri, S. (2000). Impact of uncertainty and risk aversion on price and order quantity in the newsvendor problem. Manufacturing Service Operation Management, 2, 410-423. http://dx.doi.org/10.1287/msom.2.4.410.12339

Cachon, G. (2003). Supply chain coordination with contracts. In S. Graves \& T. deKok (eds.), Handbooks in Operations Research and Management Science: Supply Chain Management (Chapter 11). Amsterdam: North-Holland.

Chen, F., \& Federgruen, A. (2000). Mean-variance analysis of basic inventory models. Working Paper, Columbia Business School, New York, USA.

Choi, T., Li, D., Yan, H., \& Chiu, C. (2008). Channel coordination in supply chains with agents having mean-variance objectives. Omega, 36, 565-76.

http://dx.doi.org/10.1016/j.omega.2006.12.003

Driessen, T.S.H. (1988). Cooperative games, solutions and applications. Boston: Kluwer Academic Publishers.

Eeckhoudt, L., Gollier, C., \& Schlesinger, H.(1995). The risk-averse(and prudent) newsboy. Management Science, 41, 786-794. http://dx.doi.org/10.1287/mnsc.41.5.786

Granot, D., \& Sosic, G. (2005). Formation of alliances in internet-based supply exchanges. Management Science, 51, 92-105. http://dx.doi.org/10.1287/mnsc.1040.0213

Guo, H.F., Leng, M.M., \& Wang, Y.I. (2012). Interchange Fee Rate, Merchant Discount Rate, and Retail Price in a Credit Card Network: A Game-Theoretic Analysis. Naval Research Logistics, 59, 525-551. http://dx.doi.org/10.1002/nav.21505

Gerchak, Y., \& Gupta. D. (1991). On apportioning costs to customers in centralized continuous review systems. Journal of Operations Management, 10, 546-551. http://dx.doi.org/10.1016/02726963(91)90010-U

Hsieh, C.C., \& Lu, Y.T. (2010). Manufacturer's return policy in a two-stage supply chain with two risk-averse retailers and random demand. European Journal of Operational Research, 207(1), 514-523. http://dx.doi.org/10.1016/j.ejor.2010.04.026

Kim, K.K., \& Park, K.S. (2014). Transferring and sharing exchange-rate risk in a risk-averse supply chain of a multinational firm. European Journal of Operational Research, 237(2), 634-648. http://dx.doi.org/10.1016/j.ejor.2014.01.067

Leng, M.M., \& Parlar, M. (2012). Transfer pricing in a multidivisional firm: A cooperative game analysis. Operations Research Letters, 40, 364-369. http://dx.doi.org/10.1016/j.orl.2012.04.009 
Ma, L., Liu, F., Li, S., \& Yan, H. (2012). Channel bargaining with risk-averse retailer. International Journal of Production Economics, 139(1), 155-167.

http://dx.doi.org/10.1016/j.ijpe.2010.08.016

Neumann, J.V., \& Morgenstern, O. (1944). Theory of Games and Economic Behaviour. Princeton: Princeton University Press.

Rosenthal, E.C. (2008). A game theoretic approach to transfer pricing in a vertically integrated supply chain. International Journal of Production Economics, 115, 542-552. http://dx.doi.org/10.1016/j.ijpe.2008.05.018

Straffin, P.D. (1993). Game theory and strategy. Washington, D.C.: The Mathematical Association of America.

Shapley, L.S. (1953). A value for n-person games. In H.W. Kuhn \& A.W. Tucker (Eds.), Contributions to the Theory of Games II. Princeton: Princeton University Press.

Shapley, L.S. (1971). Cores of convex games. International Journal of Game Theory, 1, 11-26. http://dx.doi.org/10.1007/BF01753431

Tsay, A. (2002). Risk sensitivity in distribution channel partnerships : implications for manufacturer return policies. Journal of Retailing, 78, 147-160. http://dx.doi.org/10.1016/S00224359(02)00070-2

Xu, G., Dan, B., \& Zhang, X. (2014). Coordinating a dual-channel supply chain with risk-averse under a two-way revenue sharing contract. International Journal of Production Economics, 147, Part A (0), 171-179. http://dx.doi.org/10.1016/j.ijpe.2013.09.012

Yu, G., Yang, Y., Xing, Q., \& Li, F. (2014). Research on the time optimization model algorithm of Customer Collaborative Product Innovation. Journal of Industrial Engineering and Management, 7(1), 137-152. http://dx.doi.org/10.3926/jiem.772

Article's contents are provided on a Attribution-Non Commercial 3.0 Creative commons license. Readers are allowed to copy, distribute and communicate article's contents, provided the author's and Journal of Industrial Engineering and Management's names are included. It must not be used for commercial purposes. To see the complete license contents, please visit http://creativecommons.org/licenses/by-nc/3.0/.
} 